\title{
Negative Impact of Obesity on Dialysis Efficiency: A Prospective Cohort on Lebanese CKD Patients
}

\author{
Rima Al Raii ${ }^{1} \dagger$, Mohamad Rima ${ }^{2}$, Julien Harb ${ }^{3}$, Ziad Fajloun ${ }^{4,5}$ and Katia Haddad*5 \\ ${ }^{1}$ Department of Nutrition, Lebanese University, Lebanon \\ ${ }^{2}$ IGBMC, University of Strasbourg, France \\ ${ }^{3}$ Department of Biomedical Sciences, University of Balamand, Lebanon \\ ${ }^{4}$ Laboratory of Applied Biotechnology (LBA3B), Lebanese University, Lebanon \\ ${ }^{5}$ Faculty of Sciences, Lebanese University, Lebanon \\ *Corresponding author: Katia Haddad, Department of Biology, Faculty of Sciences3, Lebanese University, Michel Slayman Tripoli \\ Campus, Ras Maska 1352, Lebanon.
}

To Cite This Article: Rima Al Raii, Mohamad Rima, Julien Harb, Ziad Fajloun, Katia Haddad, Negative Impact of Obesity on Dialysis Efficiency: A Prospective Cohort on Lebanese CKD Patients. 2020 - 11(1). AJBSR.MS.ID.001614. DOI: 10.34297/AJBSR.2020.11.001614.

Received: 制 December 12, 2020; Published: 眥 December 14, 2020

\begin{abstract}
Background: Obesity is considered as a risk factor in chronic kidney disease (CKD), as it may worsen renal complications and progress to advanced stages of the disease. Aims: To investigate whether the efficacy of dialysis is affected in obese CKD patients.

Methods: we followed the main biological factors in dialysis patients for six months. Patients were grouped based on their sexes and body mass indexes (obese, or normal-weighted) and blood samples measurements were performed before and after dialysis.

Results: The elimination of creatinine and urea by dialysis is more significant in normal-weighted than obese women. Elimination of potassium and sodium have tendency to be also more effective in normal-weighted women. No significant differences were found between normal-weighted and obese men regarding creatinine, urea, potassium, and sodium elimination. However, parathyroid hormone levels were found to be higher in obese subjects of both sexes.
\end{abstract}

Conclusion: Together these findings suggest that obesity affect dialysis efficacy.

Keywords: Obesity, BMI, Choric kidney disease, Dialysis

\section{Introduction}

For a long time, obesity was considered a simple aesthetic problem; however, today it is recognized as a real pathology and is declared by the World Health Organization as the first non-infectious epidemic in history and major problem of the century [1]. Obesity has been linked to renal diseases, as it is one of the pathologies that leads to renal failure. Therefore, physicians emphasize the importance of regular physical activity, a balanced diet, and a stable weight to protect kidneys [2,3]. These recommendations are also given for patients suffering from chronic renal failure, as healthy lifestyle is a huge help in managing chronic kidney disease (CKD) [4]. The hypothesis that obesity worsen kidney condition is con troversial. Some studies showed that obesity accelerates decline in kidney function and patient's health, while others highlighted a protective effect for dialysis patients. For example, it has been shown that obesity increases dialysis and death risk in young pre-dialysis patients [5] and reduce quality of life [6]. However, other studies concluded that body weight gain was beneficial for CKD-patients (for review, see [7]). This paradoxical relation between obesity and CKD-patients health inspired to study hemodialysis efficiency in obese and normal-weighted CKD-patients among the Lebanese population. By following creatinine, urea, sodium, potassium, phosphate, and calcium levels for 6 months, we show a decrease in the effectiveness of dialysis in obese patients. 


\section{Materials and Methods}

\section{Patients}

The study prospectively involved 40 patients with chronic renal failure that were dialyzed at hospital Alislami, Tripoli, Lebanon. Patients, aged between 45-65 years and dialyzed for 10 years, were divided in two equal groups based on their sexes. Each group was divided in two subgroups based on the patient BMI. As such, obese subgroup regrouped patients with a $30<\mathrm{BMI}<40$, while normal-weighted patients with $18.5<\mathrm{BMI}<25$ were considered as control. The study was presented to all patients and informed consent was obtained from each participant.

\section{Blood Measurements}

Blood was collected from all patients before and after their 3 weekly dialysis sessions for 6 months (March - September 2019). The main biological factors followed in dialysis patients (creatinine, urea, sodium, potassium, phosphorus, and calcium) were measured using Olympus AU480 Chemistry Analyzer (Beckman Coulter). Creatinine, urea, sodium, and potassium levels were measured 3 times/week, before and after dialysis, while phosphorus and cal- cium levels were quantified monthly and only before dialysis. For each patient, the elimination ratio of each compound was calculated using the formula below

\section{Statistics}

Data analysis was performed using Sigmaplot 11.0 statistical software. Results are presented as mean \pm SD. Statistical significance was assessed via paired t-test and ${ }^{*}$ p values $<0.05$ were considered statistically significant.

$$
\text { Elimination ratio }=\frac{\text { Value }_{\text {before dialysis }}}{\text { Value }_{\text {after dialysis }}}
$$

\section{Results}

A prospective cohort of 40 patients with chronic renal failure (including 20 women and 20 men) and undergoing dialysis was recruited. Patients were subgrouped based on their BMI, as obese or normal-weighted, and the main biological factors followed in hemodialysis were assessed over 6 months to check the possible impact of obesity on dialysis efficiency (Table 1).

Table 1: Clinical and biochemical characteristics of patients. Data are presented as mean \pm SD. $n-w$ : normal-weighted.

\begin{tabular}{|c|c|c|c|c|c|}
\hline \multicolumn{2}{|c|}{} & n-w women & obese women & n-w men & obese men \\
\hline \multirow{2}{*}{ BMI (kg/m2) } & & $20.9 \pm 2.06$ & $32.1 \pm 2.6$ & $21.4 \pm 1.69$ & $33.5 \pm 1.84$ \\
\hline \multirow{2}{*}{ Creatinine (mg/dl) } & Before & $89.6 \pm 17.1$ & $90.6 \pm 20.6$ & $98.7 \pm 26.7$ & $112.2 \pm 25.4$ \\
\cline { 2 - 6 } & After & $29.8 \pm 7$ & $36 \pm 7$ & $41.4 \pm 13.6$ & $45.2 \pm 11.3$ \\
\hline \multirow{2}{*}{ Sodium (mmol/L) } & Before & $134.2 \pm 2.5$ & $133.3 \pm 2.5$ & $134.6 \pm 1.3$ & $135.7 \pm 1.8$ \\
\cline { 2 - 6 } & After & $136 \pm 1.3$ & $135.5 \pm 1$ & $135.8 \pm 1$ & $136.2 \pm 1.5$ \\
\hline \multirow{2}{*}{ Potassium (mmol/L) } & Before & $5.8 \pm 0.5$ & $5.9 \pm 0.9$ & $5.6 \pm 1$ & $5.3 \pm 0.7$ \\
\cline { 2 - 6 } & After & $3.8 \pm 0.1$ & $1.6 \pm 0.2$ & $1.7 \pm 0.4$ & $3.8 \pm 0.5$ \\
\hline \multirow{2}{*}{ Urea (mg/dl) } & Before & $1.4 \pm 0.3$ & $0.5 \pm 0.1$ & $0.6 \pm 0.2$ & $0.7 \pm 0.1$ \\
\cline { 2 - 6 } & After & $0.3 \pm 0.1$ & 0.2 \\
\hline
\end{tabular}

Our results show that creatinine elimination ratio is significantly higher in normal-weighted women compared to obese women (Figure 1A), suggesting that obesity affect creatinine elimination efficiency by dialysis. Also, significant difference in creatinine elimination ratio was reported when comparing normal-weighted women to normal-weighted men. Urea elimination ratio was also found to be significantly higher in normal-weighted women compared to obese women, suggesting that obesity reduce urea elimination by dialysis (Figure 1B). Similarly, to creatinine, significant difference in urea elimination ratio was reported when comparing normal-weighted women to normal-weighted men. However, no significant difference was found in creatinine and urea elimination ratio between normal-weighted and obese men (Figure $1 \mathrm{~A} \&$ $1 \mathrm{~B}$, respectively), showing that obesity affected dialysis-mediated elimination of creatinine and urea only in women. For sodium and potassium elimination, no significant differences were observed between normal-weighted and obese patients (Figure 1C \& 1D, respectively). However, the elimination efficiency of both electrolytes has tendency to decrease in obese women, which is not the case when comparing between men groups (Figure $1 \mathrm{C} \& 1 \mathrm{D}$, respectively). These findings also emphasize that obesity affect dialysis efficiency (Figure 1). 

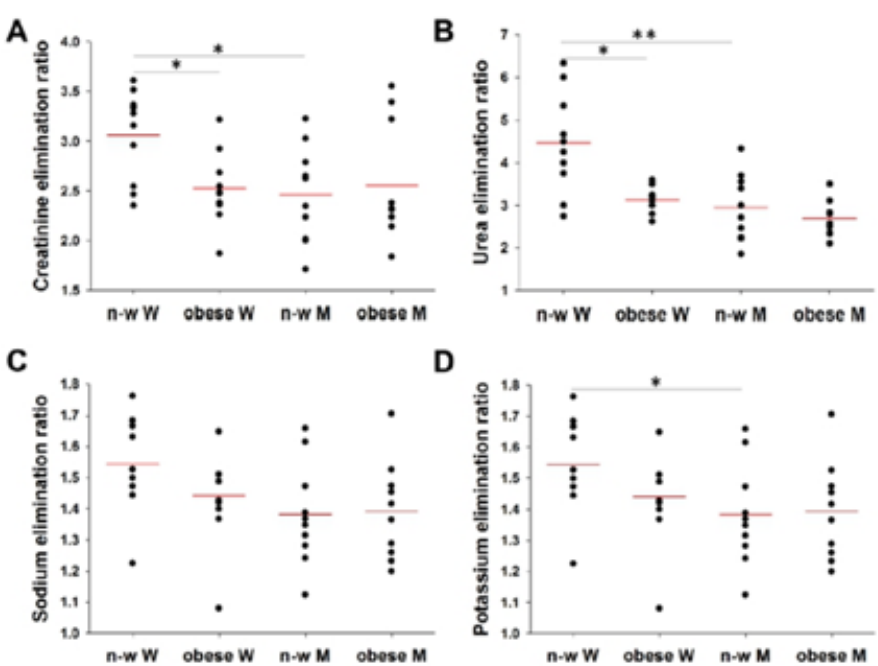

Figure 1: Elimination ratio of creatinine $(A)$, urea $(B)$, sodium $(C)$, and potassium $(D)$ by dialysis in CKD-patients. $n$-w W: normal-weighted women; Obese W: obese women; n-w M: normal-weighted men; Obese M: obese men. ${ }^{*} p<0.05,{ }^{* *} p<0.01$.

Calcium and phosphate blood levels before dialysis were followed monthly as a routinely task for each patient. Our results did not show any significant differences between obese and normal-weighted corresponding groups (Figure 2A \& 3B). However, phosphate levels had tendency to be higher in obese men, when compared to normal-weighted men. To validate this observation, we measured Parathyroid hormone (PTH) levels in all patients. The average rate of PTH in obese men $(567 \mathrm{pg} / \mathrm{mL})$ was found to be greater than that in normal-weighted men (432 pg/mL). Similarly, obese women showed higher PTH level (537 pg/ml) than normal-weighted women $(380 \mathrm{pg} / \mathrm{ml})$. Together, these results suggest that PTH metabolism, which regulates calcium and phosphate levels, is also vulnerable to obesity in dialysis patients (Figure 2).
A

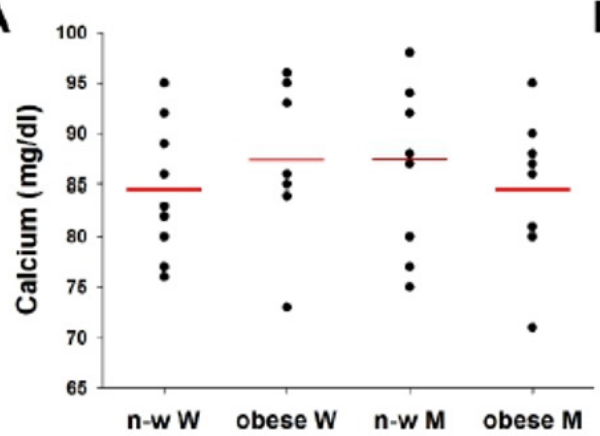

B

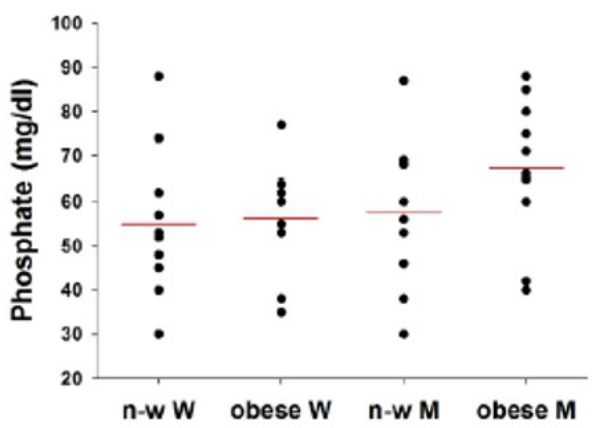

Figure 2: Calcium (A) and phosphate (B) levels in CKD-patients followed for 6 months. n-w W: normal-weighted women; obese W: obese women; n-w M: normal-weighted men; obese M: obese men.

\section{Conclusion}

Obesity is associated with accelerated decline in kidney function; therefore, is considered as one of the main risk factors for CKD $[5,8]$. Several studies investigated the effect of obesity on dialyzed CKD-patients health, but their results were contradictory. For example, it has been shown that having a BMI $>30 \mathrm{~kg} / \mathrm{m} 2$ would increase proteinuria $[9,10]$ and the glomerular filtration rate [11], while it would reduce quality of life [6]. Therefore, monitoring BMI and nutritional status of hemodialysis patients is important for their wellbeing. In agreement with these findings, it was reported that obesity in young pre-dialysis patients was one of the risk factors for initiating dialysis and death compared to those with normal BMI
[5]. Contrarily, other studies pointed to survival advantages in advanced CKD that correlates with body weight gain (for review, see [12]). Researchers suggest that the benefits of body weight gain are implicitly related to muscle mass increase, which helps in improving patient's health [13-16]. Between these two paradoxical facts, we studied the impact of obesity on hemodialysis efficiency of CKD patients, in northern Lebanon. To this end, we followed the levels of the main biological factors mentored in dialysis patients, including creatinine, sodium, potassium, and urea, for 6 months, before and after each dialysis session. Patients were subgrouped based their sexes and body mass indexes (obese, or normal-weighted) and the elimination ratio of each factor was calculated. 
Muscles use creatine for energy and creatinine production. After dialysis, creatinine levels must decrease to one third of its value before dialysis, which is equivalent to elimination ratio equal to three. This was observed in normal-weighted women, but not obese women $(30<\mathrm{BMI}<40)$, suggesting that obesity decreases the effectiveness of hemodialysis in removing creatinine from patients' blood. This was also the case for urea, potassium and sodium elimination efficiency by dialysis that was decreased in obese women. Parathyroid hormone, which regulates calcium and phosphate levels, were also increased in obese patients. Since phosphate is one of the most remarkable indicators of the progression of chronic renal failure, these findings suggest that obesity worsen kidney condition. Taken together, our results emphasize that obesity negatively affect dialysis efficiency in CKD-patients. This agrees with previous findings describing health complications observed in obese hemodialysis patients [9-11]. Interestingly, the elimination of these factors by dialysis was similar between obese $(30<\mathrm{BMI}<40)$ and normal-weighted men. Therefore, the fact that dialysis efficiency is reduced only in obese women, but not obese men may be due to muscle mass differences between the two sexes, which distort BMI values. In fact, it is known that Women have lower total muscle mass than men [17]. While caloric intake is mainly converted into muscle in men, women tend to convert calories into fat $[18,19]$. In our study, men patients (obese and normal-weighted) showed higher creatinine levels than obese and normal-weighted women respectively (Table 1), suggesting larger muscle mass in men than women. Consequently, in obese women patients, the elevated BMI may be caused by real fat mass, while in obese men patients, high BMI is probably influenced by high muscle mass. Hence, we propose to take in consideration fat and muscle mass in CKD-patients rather than their abstract BMI, as indicators for obesity. This agrees with previous findings suggesting waist-to-hip ratio as a reliable parameter that indicates central obesity [8]. To conclude, this study emphasizes that obesity negatively affect dialysis efficiency in CKD-patients. To improve the effectiveness of hemodialysis, we suggest dietary and lifestyle management for patients that increase muscle mass and reduce their fat body content.

\section{Acknowledgement}

The authors would like to thank Dr. Helene Zayat for helpful cooperation and discussion.

\section{References}

1. Lakkis JI, Weir MR (2018) Obesity and Kidney Disease. Prog Cardiovasc Dis 61(2): 157-167.
2. Praga M, Morales E (2017) The Fatty Kidney: Obesity and Renal Disease. Nephron 136(4): 273-276.

3. Abou Mrad RM, Abu Alfa AK, Ziyadeh FN (2013) Effects of weight reduction regimens and bariatric surgery on chronic kidney disease in obese patients. Am J Physiol Renal Physiol 305(5): F613-F617.

4. Câmara NOS, Iseki K, Kramer H, Liu ZH, Sharma K (2017) Kidney disease and obesity: epidemiology, mechanisms and treatment. Nat Rev Nephrol 13(3): 181-190.

5. Hoogeveen EK, Rothman KJ, Voskamp PWM, de Mutsert R, Halbesma N, et al. (2017) Obesity and risk of death or dialysis in younger and older patients on specialized pre-dialysis care. PLoS One 12(9): e0184007.

6. Zabel R, Ash S, King N, Juffs P, Bauer J (2012) Relationships between appetite and quality of life in hemodialysis patients. Appetite 59(1): 194-199.

7. Pommer W (2018) Preventive Nephrology: The Role of Obesity in Different Stages of Chronic Kidney Disease. Kidney Dis 4(4): 199-204.

8. Blaslov K, Bulum T, Duvnjak L (2015) Waist-to-height ratio is independently associated with chronic kidney disease in overweight type 2 diabetic patients. Endocr Res 40(4): 194-198.

9. Praga M, Morales E (2006) Obesity, proteinuria and progression of renal failure. Curr Opin Nephrol Hypertens 15(5): 481-486.

10. Eknoyan G (2011) Obesity and chronic kidney disease. Nefrologia 31(4): 397-403.

11. Bosma RJ, Kwakernaak AJ, van der Heide JJH, de Jong PE, Navis GJ (2007) Body mass index and glomerular hyperfiltration in renal transplant recipients: cross-sectional analysis and long-term impact. Am J Transplant 7(3): 645-652.

12. Kalantar Zadeh K, Rhee CM, Chou J, Ahmadi SF, Park J, et al. (2017) The Obesity Paradox in Kidney Disease: How to Reconcile it with Obesity Management. Kidney Int reports 2(2): 271-281.

13. Barcellos FC, Santos IS, Umpierre D, Bohlke M, Hallal PC (2015) Effects of exercise in the whole spectrum of chronic kidney disease: a systematic review. Clin Kidney J 8(6): 753-765.

14. Anding K, Bär T, Trojniak Hennig J, Kuchinke S, Krause R, et al. (2015) A structured exercise programme during haemodialysis for patients with chronic kidney disease: clinical benefit and long-term adherence. BMJ Open 5(8): e008709.

15. Jhamb M, Weiner DE (2014) Exercise to improve physical function and quality of life in CKD. Clin J Am Soc Nephrol 9(12): 2023-2034.

16. Lin TY, Peng CH, Hung SC, Tarng DC (2018) Body composition is associated with clinical outcomes in patients with non-dialysisdependent chronic kidney disease. Kidney Int 93(3): 733-740.

17. Janssen I, Heymsfield SB, Wang ZM, Ross R (2000) Skeletal muscle mass and distribution in 468 men and women aged 18-88 yr. J Appl Physiol 89(1): 81-88.

18. Blaak E (2001) Gender differences in fat metabolism. Curr Opin Clin Nutr Metab Care 4(6): 499-502.

19. Vella CA, Kravitz L, Kravitz MS (2002) Gender differences in fat metabolism. IDEA Heal Fit Source 20(10): 36-46. 